\title{
Caracterización del perfil de sensibilidad de un panel de líneas celulares para valoración de citotoxicidad in vitro
}

\author{
Claudia Jhoana León, Sandra Milena Gómez, Sandra Johanna Morantes, \\ Claudia Patricia Cordero, Fabio Ancízar
}

Grupo de Farmacogenética del Cáncer, Departamento de Farmacia, Facultad de Ciencias, Universidad Nacional de Colombia, Bogotá, D.C., Colombia.

Introducción. La valoración de la citotoxicidad in vitro de líneas celulares derivadas de tumores humanos se emplea como bioensayo preliminar para el tamizaje de productos de origen natural con potencial actividad anticancerígena.

Objetivo. Fortalecer el modelo de valoración de citotoxicidad in vitro disponible en el laboratorio, ampliando el panel de líneas celulares y caracterizando su perfil de sensibilidad a los fármacos antineoplásicos.

Materiales y métodos. Se adicionaron al panel las líneas celulares HeLa, MKN-45 y U-937, y se evaluó la sensibilidad de las siete líneas celulares (HEp-2, HT-29, MCF-7, SiHa, MKN-45, HeLa y U-937) a los fármacos antineoplásicos doxorrubicina $\mathrm{HCl}$, taxol, cisplatino, ciclofosfamida y carmustina, usados en la terapia antineoplásica. Para la valoración de la citotoxicidad se empleó el método de reducción del metil-tiazol-tetrazolio.

Resultados. Al comparar las concentraciones letales $50\left(\mathrm{CL}_{50}\right)$ calculadas, se evidenció una sensibilidad diferencial de las líneas celulares frente a doxorrubicina $\mathrm{HCl}$, taxol y cisplatino, siendo HEp-2 la línea más sensible a todos los fármacos, en tanto que las HeLa y U-937 fueron las más resistentes. La respuesta de HEp-2 frente al taxol presentó un comportamiento bifásico, relacionado con su mecanismo de acción.

Conclusión. En las condiciones empleadas no se observaron efectos frente a la ciclofosfamida y la carmustina.

Palabras clave: ensayos de selección de medicamentos antitumorales, agentes antineoplásicos, línea celular.

Sensitivity profile of a panel of cell lines designed for the evaluation of in vitro cytotoxicity

Introduction. Preliminary in vitro cytoxicity evaluations are determined in human tumor cell lines as a bioassay for the screening of potentially anticancer natural products.

Objective. To strengthen the available in vitro cytotoxicity evaluation models, the panel of cell lines was expanded, and the sensitivity profile of each cell line was evaluated for its response to selected antineoplasic drugs.

Materials and Methods. HeLa, MKN-45 and U-937 cell lines were added to the panel, and the sensitivity was determined for each of seven cell lines: HEp-2, HT-29, MCF-7, SiHa, MKN-45, $\mathrm{HeLa}$ and U-937. The effects of the antineoplasic drugs Doxorubicin HCl, Taxol, Cisplatin, Cyclophosphamide and Carmustin were examined, using the methyl thiazol tetrazolium (MTT) reduction assay.

Results. A differential sensitivity to the drugs Doxorubicin $\mathrm{HCl}$, Taxol and Cisplatin was established among the cell lines by comparing the lethal concentration 50 (LC50) values. HEp2 was the most sensitive cell line, whereas HeLa and U-937 were the most resistant. HEp-2 exhibited a biphasic response to Taxol treatment; this was related to the reported mechanism of action of this compound.

Conclusion. Cyclophosphamide and Carmustin did not show activity under test conditions.

Keywords: drug screening assays, antitumor, antineoplastic agents, cell line. 
La búsqueda de nuevas moléculas con potencial actividad anticancerígena a partir de productos naturales es hoy en día un campo vigente $(1,2)$. En este proceso, la valoración de la citotoxicidad in vitro sigue siendo una herramienta válida y útil en las primeras etapas de selección de compuestos promisorios, que los grupos de investigación básica utilizan ampliamente alrededor del mundo (3-6).

Para dar solidez a las valoraciones es necesario disponer de un panel de líneas celulares que represente diversos tipos de tumores, y conocer su sensibilidad a agentes con acción citotóxica demostrada.

Para la selección de productos con potencial actividad anticancerígena, el Instituto Nacional del Cáncer de los Estados Unidos estableció en 1989 el tamizaje de líneas celulares, y, actualmente, emplea un panel de 60 líneas que representan nueve tipos de tumores humanos (cerebro, colon, pulmón, ovario, riñón, melanoma, leucemia, mama y próstata) (7).

Con estas líneas se ha evaluado una serie de agentes antineoplásicos con diferentes mecanismos de acción, generando perfiles de sensibilidad frente a los cuales se puede comparar el perfil de nuevas sustancias en estudio, lo que permite plantear hipótesis sobre su mecanismo de acción y sus blancos moleculares (8).

A lo largo de los años, el modelo ha mostrado buena correlación entre el patrón de actividad de un compuesto y el panel de células y su mecanismo de acción, generando información útil en el desarrollo de nuevos agentes anticancerígenos $(7,9)$.

Desde 1999, el Instituto Nacional del Cáncer de los Estados Unidos realiza y recomienda a otros laboratorios iniciar el tamizaje de un reducido panel de líneas celulares altamente sensibles y sólo

\footnotetext{
Correspondencia:

Fabio Ancízar Aristizábal, Carrera 30 № 45-03, Edificio 450, Oficina 206, Ciudad Universitaria, Bogotá D.C.

Teléfono (571) 3165120, Fax (571) 3165060

faaristizabalg@unal.edu.co

Recibido: 05/08/05; aceptado: 16/12/05
}

evaluar con el panel amplio, los productos que muestren actividad a este nivel $(2,7)$.

En nuestro laboratorio se estableció el modelo de valoración de citotoxicidad con cuatro líneas celulares (10), dos de ellas incluidas en el panel del Instituto Nacional de Cáncer de los Estados Unidos (MCF-7 y HT-29). Todas fueron caracterizadas de acuerdo con su sensibilidad frente al agente citotóxico doxorrubicina $\mathrm{HCl}$, empleado como control positivo de actividad en las valoraciones realizadas. Con el fin de fortalecer el modelo, en este trabajo se amplió el número de tipos tumorales representados en el panel de trabajo, y se incluyeron dos líneas donadas por el Instituto Nacional de Cancerología de Colombia y una por la Fundación Instituto de Inmunología de Colombia. Además, se caracterizó la sensibilidad de todas las líneas utilizando una serie de agentes antitumorales de uso clínico bien conocidos, que presentan diferentes mecanismos de acción.

\section{Materiales y métodos}

\section{Líneas celulares}

Se emplearon las líneas celulares derivadas de tumores sólidos humanos MCF-7 (adenocarcinoma de mama), HEp-2 (carcinoma de laringe), HT-29 (adenocarcinoma colorrectal), HeLa y SiHa (carcinoma de cuello uterino), MKN-45 (carcinoma gástrico), obtenidas del banco de células del Laboratorio de Inmunología del Instituto Nacional de Cancerología de Colombia, y U-937 (adherente derivada de linfoma histiocítico), obtenida de la Fundación Instituto de Inmunología. Las líneas celulares se mantuvieron en frascos de cultivo de $75 \mathrm{~cm}^{2}$ de área, con medio mínimo esencial y suplemento con $5 \%$ de suero fetal bovino, penicilina, $100 \mathrm{Ul} / \mathrm{mL}$ y estreptomicina, $100 \mu \mathrm{g} /$ $\mathrm{mL}$. Se incubaron a $37^{\circ} \mathrm{C}$ en atmósfera de $5 \%$ de $\mathrm{CO}_{2}$ y $100 \%$ de humedad relativa.

Los cultivos celulares con $90 \%$ de confluencia se trataron con solución de tripsina al 0,025\% y EDTA al $0,03 \%$ durante 5 minutos a $37^{\circ} \mathrm{C}$, y se obtuvo una suspensión celular que se contó en cámara de Neubauer mediante el método de exclusión de azul de tripano. A cada pozo de las placas de microtitulación (de 96 pozos) y de fondo plano, se transfirieron $100 \mathrm{~mL}$ de suspensión celular con 
la densidad celular establecida para cada línea. Las placas se incubaron durante 24 horas antes de cualquier manipulación para así permitir la adhesión de las células a la superficie del pozo.

Para las líneas celulares MKN-45, HeLa y U-937, se dispuso por triplicado un número creciente de células por pozo: $0,500,1.000,2.500,5.000$, $10.000,15.000,20.000,25.000$ y 30.000 en un volumen constante de $100 \mu \mathrm{L} /$ pozo. Al cabo de 24 horas de incubación se realizó un cambio de medio, se adicionaron $100 \mu \mathrm{L}$ de medio fresco a cada pozo, y se incubó durante 48 horas. Al cabo de este tiempo se determinó la absorbancia empleando el método de reducción del metiltiazol- tetrazolio, para establecer el rango en que la relación entre la densidad celular y la absorbancia mantenían una relación lineal. La determinación se realizó en dos ensayos en semanas diferentes.

El método de reducción de la sal de tetrazolio bromuro de 3-(4,5-dimetiltiazol-2-il)-2,5-difeniltetrazolio, conocida como metil-tiazol- tetrazolio, es una medida del metabolismo celular basada en la reducción de esta sal por acción de las succinato-deshidrogenasas mitocondriales, con la consecuente formación del colorante azul-violeta formazán, cuya producción es proporcional al número de células metabólicamente activas (11).

Para la aplicación de este método se siguió el procedimiento establecido por Mosmann (11), adaptado para nuestro laboratorio por Cordero y Aristizábal (10) con las siguientes modificaciones: al terminar el tratamiento se retiró el medio de cultivo metabolizado (para evitar que el pH bajo y el porcentaje de glucosa bajo interfirieran con el metabolismo de la sal de tetrazolio); se lavó cada pozo con solución salina tamponada con fosfatos, y se adicionó una solución de metil-tiazol-tetrazolio de $0,25 \mathrm{mg} / \mathrm{mL}$ recién preparada a medio de cultivo sin suplementar. Se incubó a $37^{\circ} \mathrm{C}$ durante 4 horas y se retiró el sobrenadante, cuidando de no arrastrar los cristales de formazán. Los cristales se disolvieron en dimetilsulfóxido. Las placas se agitaron por 5 minutos en agitador de placas a 800 revoluciones por minuto y temperatura ambiente, y se leyeron a $570 \mathrm{~nm}$ en el lector de placas BIORAD 550.

\section{Ensayos de citotoxicidad}

Se emplearon las formas comerciales de los fármacos doxorrubicina $\mathrm{HCl}$ (Pharmacia \& Upjohn), cisplatino, ciclofosfamida, taxol y carmustina (Bristol Myers Squib) en cinco diluciones seriadas de 1:10, con una concentración máxima de 10 $\mu \mathrm{M}(10$ a $5 \mathrm{M})$. Se realizó una evaluación preliminar para seleccionar el rango de concentraciones de trabajo para cada fármaco con el fin de determinar la $\mathrm{CL}_{50}$.

Las células cultivadas en placas con la densidad de trabajo seleccionada para cada línea, se trataron durante 48 horas con la sustancia de prueba en cinco diluciones seriadas (tres réplicas o pozos con cada dilución), dejando en la placa pozos con células sin tratar como controles de crecimiento, y pozos sin células, pero con tratamiento como blanco. Después del tratamiento se determinó la población celular viable mediante el método de reducción del metil-tiazol-tetrazolio, se calcularon los porcentajes de supervivencia celular relativos a las células control de crecimiento y se construyeron curvas de concentración versus porcentaje de supervivencia. Con base en éstas, se calcularon las concentraciones que inducían la muerte al $50 \%$ de las células tratadas, o concentraciones letales 50 . Se realizaron tres réplicas en semanas diferentes. En las valoraciones no se empleó un período de recuperación posterior al tratamiento, razón por la cual la citotoxicidad se reporta en términos de concentración letal 50 (12).

\section{Métodos estadísticos}

Se aplicó una prueba de paralelismo $t$-Student para comparar las dos réplicas realizadas con el fin de establecer el rango de linealidad entre la absorbancia y la densidad celular de las células que se iban a incluir en el panel, esto con el fin de determinar la reproducibilidad del resultado obtenido.

Los resultados de la actividad citotóxica de los fármacos sobre el panel de líneas celulares se analizaron empleando un análisis de varianza (ANOVA), para establecer la influencia de los factores de tratamiento y línea celular en el resultado de $\mathrm{CL}_{50}$ obtenido. 


\section{Resultados}

Determinación del rango de linealidad entre absorbancia y densidad celular para MKN-45, HeLa y U-937

Para las líneas celulares que iban a ser adicionadas al panel de trabajo, se evaluó la linealidad entre la absorbancia y la densidad celular (número de células/pozo). El rango en unidades de absorbancia en que se observó una relación lineal fue de 0,06 a 1,9 para MKN-45, 0,06 a 1,2 para U-937 y 0,06 a 1,6 para HeLa (figura 1). Esta determinación se realizó por duplicado y no se observó diferencia significativa entre las dos rectas obtenidas para cada línea celular al aplicar una prueba de paralelismo $(p>0,05)$.

Dentro del rango de linealidad determinado, para cada línea se calculó la densidad celular correspondiente a un valor de absorbancia cercano a la unidad de $570 \mathrm{~nm}$, que en adelante se empleó como la densidad celular de trabajo. Estas densidades fueron de 15.000 células/pozo para MKN-45 y de 10.000 células/pozo para HeLa y U937. Con microscopio invertido se comprobó visualmente que con estas densidades los pozos presentaban un estado de confluencia cercano al $90 \% 72$ horas después de la inoculación (24 horas de incubación previa a la adición del fármaco más 48 horas de incubación), para evitar variación de

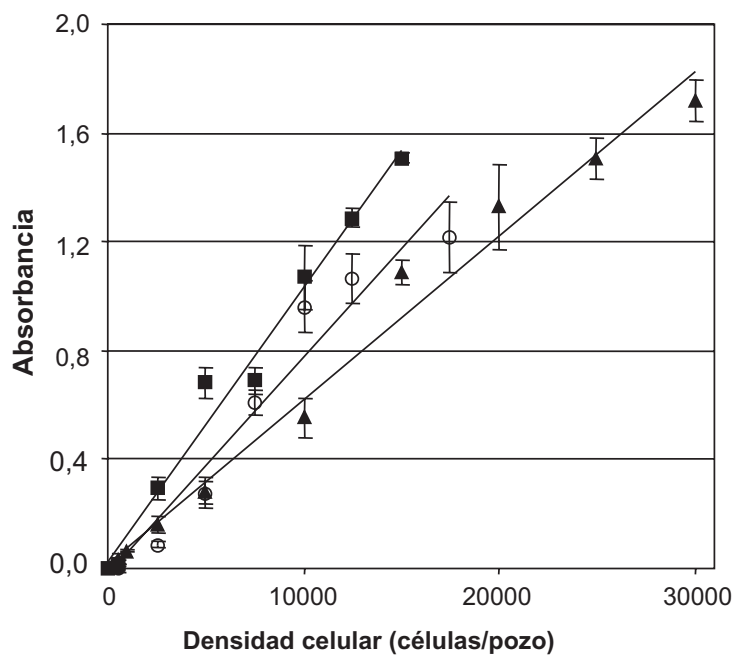

Figura 1. Determinación del rango de linealidad, $\square$ MKN-45, o U-937 y $\mathrm{H}$ HeLa. Se presenta sólo una de las réplicas para cada línea celular. Absorbancia medida a $570 \mathrm{~nm}$. los resultados por desprendimiento de la monocapa celular.

Para las demás líneas celulares se emplearon las densidades de trabajo establecidas previamente: 7.500 células/pozo para HEp-2, 10.000 células/ pozo para SiHa, 15.000 células/pozo para MCF-7 y 20.000 células/pozo para HT-29 (10).

\section{Evaluación de la sensibilidad de las líneas celulares frente a fármacos antineoplásicos}

Se emplearon los fármacos carmustina, cisplatino, taxol, ciclofosfamida y doxorrubicina $\mathrm{HCl}$. Las siete líneas celulares empleadas (MCF7, HEp-2, HT-29, HeLa, SiHa, MKN-45 y U-937) resultaron sensibles a cisplatino, taxol y doxorrubicina $\mathrm{HCl}$, mientras que no mostraron sensibilidad a carmustina ni a ciclofosfamida. En el cuadro 1 y en la figura 2 se presentan los valores de las $\mathrm{CL}_{50}$ calculadas para la doxorrubicina $\mathrm{HCl}$, el cisplatino y el taxol.

\section{Sensibilidad a taxol, doxorrubicina $\mathrm{HCl}$ y cisplatino}

En la valoración preliminar de las líneas celulares HEp-2 y HT-29 con taxol (concentraciones entre $10-9 \mathrm{M}$ y $10-5 \mathrm{M}$ ), se presentó un descenso de la respuesta, o sea, el porcentaje de superviviencia, con concentraciones bajas, una estabilización o meseta en las concentraciones medias y, luego, un nuevo descenso en las concentraciones altas (figura 3). Para el cálculo de la $\mathrm{CL}_{50}$, se empleó la zona anterior a la fase de meseta (concentraciones bajas).

Las líneas celulares fueron más sensibles al taxol que a la doxorrubicina $\mathrm{HCl}$ y al cisplatino,

Cuadro 1. Concentraciones letales 50 calculadas*

\begin{tabular}{lccr}
\hline & \multicolumn{3}{c}{ Fármaco } \\
\cline { 2 - 4 } $\begin{array}{l}\text { Línea } \\
\text { celular }\end{array}$ & $\begin{array}{c}\text { Taxol } \\
(\mu \mathbf{M})\end{array}$ & $\begin{array}{c}\text { Doxo } \mathbf{~ H C l} \\
(\mu \mathbf{M})\end{array}$ & $\begin{array}{c}\text { Cisplatino } \\
(\mu \mathbf{M})\end{array}$ \\
\hline HeLa & $9,9 \pm 1,7$ & $1,63 \pm 0,35$ & $15,9 \pm 5,6$ \\
U-937 & $9,7 \pm 0,8$ & $0,22 \pm 0,05$ & $14,2 \pm 1,8$ \\
MKN-45 & $1,21 \pm 0,14$ & $1,61 \pm 0,1$ & $9,2 \pm 0,4$ \\
SiHa & $0,9 \pm 0,3$ & $0,23 \pm 0,06$ & $19,1 \pm 5,9$ \\
MCF-7 & $0,76 \pm 0,05$ & $0,19 \pm 0,06$ & $16,4 \pm 2,8$ \\
HT-29 & $0,025 \pm 0,002$ & $1,07 \pm 0,34$ & $19,4 \pm 6,5$ \\
HEp-2 & $0,012 \pm 0,002$ & $0,26 \pm 0,03$ & $6,6 \pm 0,9$ \\
\hline${ }^{*} \mathrm{CL}_{50}$ media de tres determinaciones \pm desviación estándar
\end{tabular}




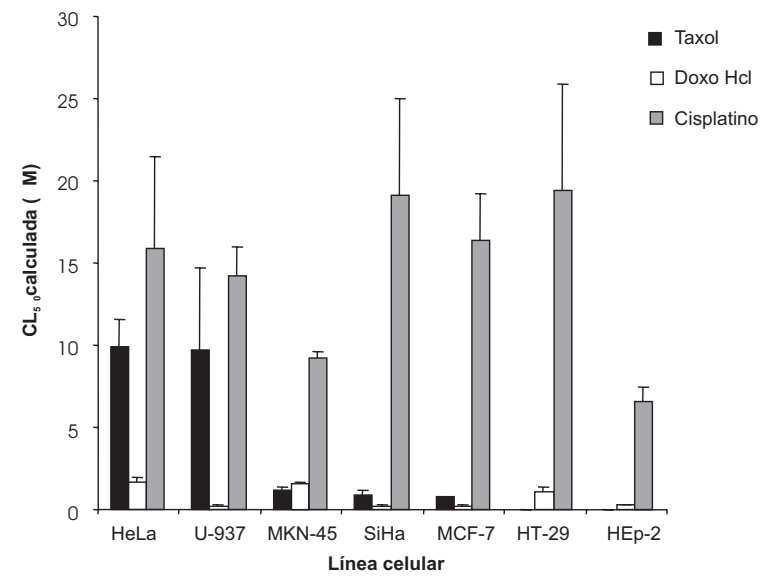

Figura 2. Concentraciones letales 50 calculadas $\left(\mathrm{CL}_{50}\right)$ para las siete líneas celulares. Valor medio de tres determinaciones, más o menos la desviación estándar.

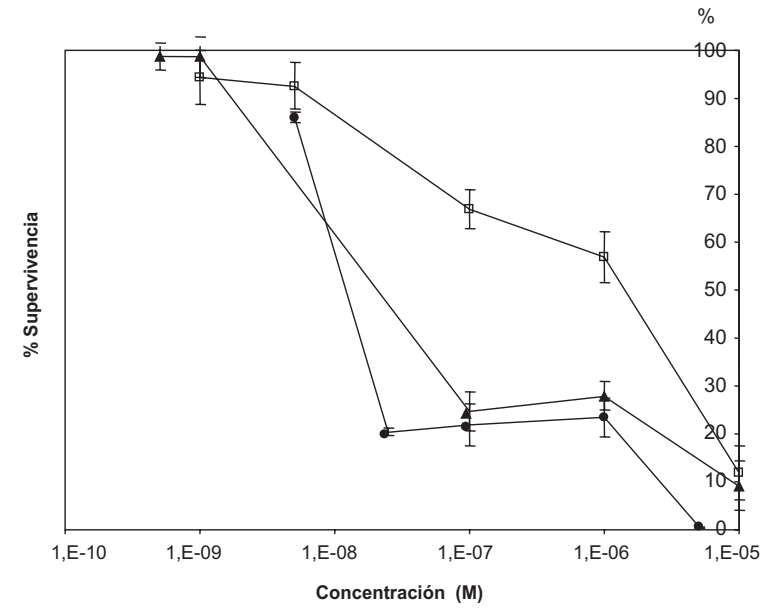

Figura 3. Evaluación preliminar de la sensibilidad a taxol, concentraciones de $5 \times 10^{-10} \mathrm{M}$ a $1 \times 10^{-5} \mathrm{M}$. प HEp-2, $\mathrm{C}$ MCF-7, 口 HT-29.

presentando el siguiente orden de sensibilidad: $\mathrm{HeLa}<\mathrm{U}-937<\mathrm{MKN}-45<\mathrm{SiHa}<\mathrm{MCF}-7<\mathrm{HT}-$ $29<$ HEp-2.

Frente al cisplatino, la HEp-2 también fue la línea celular más sensible, seguida de MKN-45. Las líneas celulares U-937 y HeLa fueron las menos sensibles a los tres fármacos.

\section{Análisis de varianza}

En el análisis de varianza (ANOVA) se consideró la contribución de las variables referentes a compuesto y línea celular a la variación de los valores de concentración letal $50\left(\mathrm{CL}_{50}\right)$ obtenidos.
El resultado mostró que tanto el factor de compuesto, como el factor de línea celular eran significativos ( $p=0.0001)$, y el factor de compuesto fue el que más contribuyó en la variación de la respuesta $\left(\mathrm{CL}_{50}\right)$. Esto se puede asociar con los diferentes mecanismos de acción que presentan los fármacos evaluados, lo que implica diferencias en su forma de interacción con cada línea celular. La significación del factor de línea celular confirma que las líneas celulares presentan un perfil de sensibilidad diferencial que posiblemente obedece a diferencias en sus características bioquímicas.

\section{Discusión}

Determinación del rango de linealidad entre absorbancia y densidad celular, para MKN-45, HeLa y U-937

En el método de reducción del metil-tiazoltetrazolio (MTT) se realiza una medición colorimétrica, en la cual la absorbancia es una medida indirecta del número de células viables; por esto, para cada línea celular que se va a emplear es necesario establecer la capacidad de reducir el MTT y producir el formazán, así como el rango de densidad celular en que se presenta una relación lineal entre la absorbancia y el número de células viables en el pozo. Para las líneas MKN-45, HeLa y U-937, que se iban a incluir en el panel de trabajo, se presentó una relación lineal desde absorbancias bajas (0,06 unidades) hasta absorbancias cercanas a dos unidades, equivalentes a pocas células y a un estado de confluencia en el pozo.

Las densidades de trabajo calculadas para estas líneas son del mismo orden de las calculadas para las otras líneas del panel, lo que permite comparar directamente los resultados obtenidos entre las siete líneas.

Estos resultados indican que las líneas pueden incluirse en el panel de trabajo.

\section{Evaluación de la sensibilidad de las líneas celulares frente a fármacos antineoplásicos}

Sensibilidad a agentes alquilantes: ciclofosfamida, carmustina y cisplatino

Las líneas celulares no mostraron ser sensibles a la ciclofosfamida ni a la carmustina. La 
ciclofosfamida es un profármaco que debe ser metabolizado por enzimas del citocromo P-450 para activarse (13); por tanto, la falta de sensibilidad sugiere una posible pérdida de la expresión de estas enzimas en las líneas celulares de trabajo, tal como lo han reportado otros autores (14), o bien una posible sobreexpresión de enzimas involucradas en la conversión de la ciclofosfamida a metabolitos inactivos, como la aldehído deshidrogenasa clase 1 (ALD-1) y la glucosa-6-fosfato deshidrogenasa (G6PD) $(15,16)$. El estudio de estos cambios de expresión está fuera de los objetivos del presente trabajo.

De otro lado, la falta de sensibilidad de las líneas celulares a la carmustina puede explicarse por la sobreexpresión de enzimas de reparación del ADN, como la O6-alquilguanina-ADN-transferasa (AGT), también reportada por otros autores (17).

\section{Sensibilidad al taxol}

La respuesta bifásica de HEp-2 y HT-29 frente al taxol se asemeja a los resultados reportados por Yeung y colaboradores (18), según los cuales el taxol induce un decrecimiento bifásico de la supervivencia celular, con una fase de meseta. Este comportamiento se atribuye a dos fenómenos dependientes de la concentración: en concentraciones bajas, el taxol estabiliza los microtúbulos del huso mitótico bloqueando la mitosis y llevando a la apoptosis; y en concentraciones altas, induce la polimerización de los microtúbulos y la formación de paquetes que inhiben la entrada a la fase $S$, induciendo necrosis.

La similitud de las curvas obtenidas para HT-29 y HEp-2 con las descritas por estos autores permite sugerir que sobre estas dos líneas celulares el taxol ejerce su acción involucrando los procesos de apoptosis o necrosis, dependiendo de la concentración.

Es de resaltar que Yeung y colaboradores (18) también encontraron tal comportamiento con fase de meseta para la línea MCF-7, el cual no se observó muy pronunciado en el presente trabajo.

Con los perfiles de sensibilidad generados para las líneas celulares incluidas en el panel se encontró que éstas responden de manera diferencial, presentando valores de $\mathrm{CL}_{50}$ de diferente orden de magnitud frente a fármacos con diferente mecanismo de acción: taxol, cisplatino y doxorrubicina $\mathrm{HCl}$.

El taxol es un agente antimitótico por su efecto estabilizador e inductor de polimerización de los microtúbulos del huso mitótico (18); la doxorrubicina $\mathrm{HCl}$ inhibe la topoisomerasa II al inducir la formación de complejos entre esta enzima y el ADN, además de intercalarse en el ADN (19), en tanto que el cisplatino es un agente alquilante (20).

La selección de compuestos de origen natural con actividad citotóxica promisoria hasta ahora se venía realizando en nuestro laboratorio, por comparación de la $\mathrm{CL}_{50}$ calculada para el compuesto frente a los valores de concentración letal 50 obtenidos para la doxorrubicina $\mathrm{HCl}$ y un valor límite de referencia de $4 \mu \mathrm{g} / \mathrm{mL}$, sugerido para compuestos puros por el Instituto Nacional del Cáncer de los Estados Unidos (21) y empleado por grupos de Latinoamérica y Europa $(22,23)$.

Con el establecimiento de los perfiles de sensibilidad para las líneas celulares incluidas en el panel de trabajo se generaron valores propios de referencia, aplicables en la selección de compuestos con potencial actividad anticancerígena, lo que le da mayor fortaleza al modelo de citotoxicidad in vitro.

De otro lado, la falta de sensibilidad de las líneas celulares frente a la ciclofosfamida sugiere que el modelo presenta limitaciones en la detección de compuestos citotóxicos que, como este fármaco, requieran una activación enzimática previa a su interacción con las células, y plantea la alternativa de complementar el estudio de citotoxicidad con un paso de activación previa por medio del tratamiento con enzimas microsómicas, mediante fracción S9, fracción microsómica u otros preparados hepáticos (24).

\section{Agradecimientos}

Al Laboratorio Bristol Myers-Squib por la donación de los compuestos antineoplásicos evaluados. Al Grupo de Investigación en Productos Bioactivos de Plantas Medicinales Colombianas del Departamento de Farmacia de la Universidad Nacional de Colombia por su constante apoyo. 


\section{Conflicto de intereses}

Los autores declaran que no tienen intereses particulares que pudieran influenciar los resultados presentados.

\section{Financiación}

Este trabajo contó con financiación de la División de Investigación Bogotá, de la Dirección Nacional de Investigación (DINAIN) de la Universidad Nacional de Colombia.

\section{Referencias}

1. Cordell GA. Biodiversity and drug discovery - a symbiotic relationship. Phytochemistry 2000;55:463-80.

2. Mans DR, da Rocha AB, Schwartsmann G. Anticancer drug discovery and development in Brazil: targeted plant collection as a rational strategy to acquire candidate anti-cancer compounds. Oncologist 2000;5:185-98.

3. Andrighetti-Fröhner CR, Antonio RV, CreczynskiPasa TB, Barardi CR, Simões CM. Cytotoxicity and potential antiviral evaluation of violacein produced by Chromobacterium violaceum. Mem Inst Oswaldo Cruz 2003;98:843-8.

4. Zhang XY, Li WG, Wu YJ, Zheng TZ, Li W, Qu SY et al. Proanthocyanidin from grape seeds potentiates antitumor activity of doxorubicin via immunomodulatory mechanism. Int Immunopharmacol 2005;5:1247-57.

5. Morales A, Pérez $\mathbf{P}$, Mendoza R, Compagnone R, Suárez Al, Arvelo $\mathbf{F}$ et al. Cytotoxic and proapoptotic activity of ent-16â-17á-dihydroxykaurane on human mammary carcinoma cell line MCF-7. Cancer Lett 2005;218:109-16.

6. Fricker SP, Buckley RG. Comparison of two colorimetric assays as cytotoxicity end point for an in vitro screen for antitumor agents. Anticancer Res 1996;16:3755-60.

7. Takimoto $\mathbf{C H}$. Anticancer drug development at the US National Cancer Institute. Cancer Chemother Pharmacol 2003;52(Suppl.1):29-33.

8. Sausville EA, Johnson Jl. Molecules for the millennium: How will they look? New Drug Discovery year 2000. Br J Cancer 2000;83:1401-4.

9. Yamori T. Panel of human cancer cell lines provides valuable database for drug discovery and bioinformatics. Cancer Chemother Pharmacol 2003; 52 (Suppl.1):74-9.

10. Cordero CP, Aristizábal FA. Evaluación preliminar in vitro de citotoxicidad de extractos vegetales, empleando métodos colorimétricos. Revista Colombiana de Biotecnología 2002;4:100-6.
11. Mosmann T. Rapid colorimetric assay for cellular growth and survival: application to proliferation and citotoxicity assays. J Immunol Methods 1983;65:5563.

12. Freshney RI. Culture of animal cells: a manual of basic technique. 4th ed. New York: John Wiley and Sons Inc; 2000. p.330.

13. Gut I, Danielová V, Holubová J, Souèek P, Kluèková H. Cytotoxicity of cyclophosphamide, paclitaxel and docetaxel for tumor cell lines in vitro: effects of concentration, time and cytochrome P450-catalyzed metabolism. Arch Toxicol 2000;74:437-46.

14. MacFadyen M, McLeod H, Jackson FC, Melvin WT, Doehmer J, Murray Gl. Cytochrome P450 CYP1B1 protein expression: a novel mechanism of anticancer drug resistance. Biochem Pharmacol 2001;62:207-12.

15. Tsukamoto N, Chen J, Yoshida A. Enhanced expressions of glucose-6-phosphate dehydrogenase and cytosolic aldehyde dehydrogenase and elevation of reduced glutathione level in cyclophosphamide resistant human leukemia cells. Blood Cells Mol Dis 1998;24:231-8.

16. Moreb J, Maccow C, Schweder M, Hecomovich J. Expression of antisense RNA to aldehyde dehydrogenase class-1 sensitizes tumor cells to 4hydroperorycyclophosphamide in vitro. J Pharmacol Exp Ther 2000;293:390-6.

17. Heim MM, Eberhardt W, Seeber S, Müller MR. Differential modulation of chemosensitivity to alkylating agents and platinum compounds by DNA repair modulators in human lung cancer cell lines. J Cancer Res Clin Oncol 2000;126:198-204.

18. Yeung TK, Germond C, Chen X, Wang Z. The mode of action of Taxol: apoptosis at low concentration and necrosis at high concentration. Biochem Biophys Res Commun 1999;263:398-404.

19. Stewart CF, Ratain MJ. Cancer topoisomerase interactive agents. In: DeVita VT, Hellman S, Rosenberg SA, editors. Principles and Practice of Oncology. 6th ed. Philadelphia: Lippincott Williams \& Wilkins; 2001. (Libro en CD-ROM).

20. Chabner B, Allegra C, Curt G, Calabresi P. Quimioterapia de las enfermedades neoplásicas. En: Hardman J, Limbird L, Molinoff P, Ruddon R, Goodman A, editores. Las bases farmacológicas de la terapéutica. México, D.F: McGraw Hill Interamericana; 1996. p.130969.

21. Geran RI, Greenberg NH, MacDonald MM, Schumacher AM, Abbott, BJ. Protocols for screening chemical agents and natural products against animal tumours and other biological systems. Cancer Chemother Rep 1972;3:1-103.

22. Betancur-Galvis L, Zuluaga C, Arnó M, González MA, Zaragoza RJ. Structure-activity relationship of in 
vitro antiviral and cytotoxic activity of semisynthetic analogues of scopadulane diterpenes. J Nat Prod 2001;64:1318-21.

23. Jantova S, Cipak L, Cernakova M, Kost'alova D. Effect of berberine on proliferation, cell cycle and apoptosis in HeLa and L1210 cells. J Pharm Pharmacol 2003;55:1143-9.
24. Brandon EF, Raap CD, Meijerman I, Beijnen JH, Schellens $\mathbf{J H}$. An update on in vitro test methods in human hepatic drug biotransformation research: pros and cons. Toxicol Appl Pharmacol 2003;189:233-46.

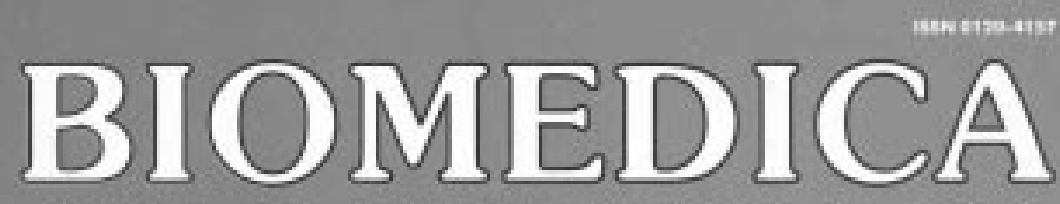

Revista det Instituto Nacionat de salud

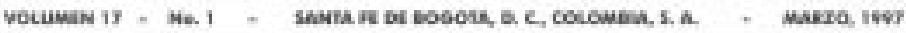
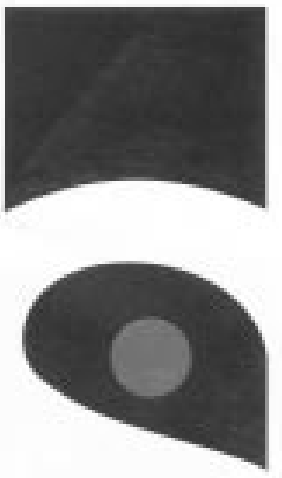

INSTITUTO

NACIONAL DE

SALUD

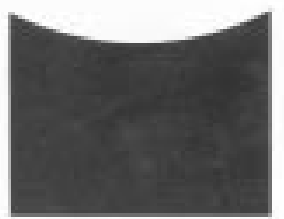

$1917-1997$ 